\title{
IDEAL CONVERGENT SEQUENCE SPACES WITH RESPECT TO INVARIANT MEAN AND A MUSIELAK-ORLICZ FUNCTION OVER $n$-NORMED SPACES
}

\author{
SUNIL K. SHARMA* \\ Department of Mathematics, Model Institute of Engineering \& Technology, \\ Kot Bhalwal 181122, J \& K, INDIA \\ *Corresponding author: sunilksharma42@gmail.com
}

\begin{abstract}
In the present paper we defined $\mathcal{I}$-convergent sequence spaces with respect to invariant mean and a Musielak-Orlicz function $\mathcal{M}=\left(M_{k}\right)$ over $n$-normed spaces. We also make an effort to study some topological properties and prove some inclusion relation between these spaces.
\end{abstract}

\section{INTRODUCTION AND PRELIMINARIES}

Let $\sigma$ be an injective mapping from the set of the positive integers to itself such that $\sigma^{p}(n) \neq n$ for all positive integers $n$ and $p$, where $\sigma^{p}(n)=\sigma\left(\sigma^{p-1}(n)\right)$. An invariant mean or a $\sigma$-mean is a continuous linear functional defined on the space $\ell_{\infty}$ such that for all $x=\left(x_{n}\right) \in \ell_{\infty}$ :

(1) If $x_{n} \geq 0$ for all $n$, then $\phi(x) \geq 0$,

(2) $\phi(e)=1$,

(3) $\phi(S x)=\phi(x)$, where $S x=\left(x_{\sigma(n)}\right)$.

$V_{\sigma}$ denotes the set of bounded sequences all of whose invariant means are equal which is also called as the space of $\sigma$-convergent sequences. In [26], it is defined by

$$
V_{\sigma}=\left\{x \in \ell_{\infty}: \lim _{k} t_{k n}(x)=\ell, \quad \text { uniformly in } n, \ell=\sigma-\lim x\right\},
$$

Received 2017-09-21; accepted 2017-12-07; published 2018-11-02.

2010 Mathematics Subject Classification. 40A05,40A35, 46A45.

Key words and phrases. $\mathcal{I}$-convergent, invariant mean, Orlicz function, Musielak-Orlicz function, $n$-normed space, $A$ transform.

(C)2018 Authors retain the copyrights of their papers, and all open access articles are distributed under the terms of the Creative Commons Attribution License. 
where $t_{k n}(x)=\frac{x_{n}+x_{\sigma^{1}(n)}+\cdots+x_{\sigma^{k}(n)}}{k+1}$.

$\sigma$-mean is called a Banach limit if $\sigma$ is the translation mapping $n \rightarrow n+1$. In this case, $V_{\sigma}$ becomes the set of almost convergent sequences which is denoted by $\hat{c}$ and defined in [11] as

$$
\hat{c}=\left\{x \in \ell_{\infty}: \lim _{k} d_{k n}(x) \text { exists uniformly in } n\right\}
$$

where $d_{k n}(x)=\frac{x_{n}+x_{n+1}+\cdots+x_{n+k}}{k+1}$.

The space of strongly almost converegnt sequences was introduced by Maddox [12] as follow:

$$
\hat{c}=\left\{x \in \ell_{\infty}: \lim _{k} d_{k n}(|x-\ell e|) \text { exists uniformly in } n \text { for some } \ell\right\}
$$

The notion of ideal convergence was first introduced by P. Kostyrko [8] as a generalization of statistical convergence which was further studied in topological spaces by Das, Kostyrko, Wilczynski and Malik see [1]. More applications of ideals can be seen in ([1], [2]). Mursaleen and Sharma [19] continue in this direction and introduced $I$-convergence of generalized sequences with respect to Musielak-Orlicz function.

A family $\mathcal{I} \subset 2^{X}$ of subsets of a non empty set $X$ is said to be an ideal in $X$ if

(1) $\phi \in \mathcal{I}$

(2) $A, B \in \mathcal{I}$ imply $A \cup B \in \mathcal{I}$

(3) $A \in \mathcal{I}, B \subset A$ imply $B \in \mathcal{I}$,

while an admissible ideal $\mathcal{I}$ of $X$ further satisfies $\{x\} \in \mathcal{I}$ for each $x \in X$ see [8].

A sequence $\left(x_{n}\right)_{n \in \mathbb{N}}$ in $X$ is said to be $\mathcal{I}$-convergent to $x \in X$, if for each $\epsilon>0$ the set $A(\epsilon)=\{n \in \mathbb{N}$ : $\left.\left\|x_{n}-x\right\| \geq \epsilon\right\}$ belongs to $\mathcal{I}$.

A sequence $\left(x_{n}\right)_{n \in \mathbb{N}}$ in $X$ is said to be $\mathcal{I}$-bounded to $x \in X$ if there exists an $K>0$ such that $\{n \in \mathbb{N}$ : $\left.\left|x_{n}\right|>K\right\} \in \mathcal{I}$. For more details about ideal convergence sequence spaces (see [7], [9], [15], [16], [17], [18], $[21],[25],[26],[27])$ and references therein.

Let $A=A_{i j}$ be an infinite matrix of complex numbers $a_{i j}$, where $i, j, \in \mathbb{N}$. We write $A x=\left(A_{i}(x)\right)$ if $A_{i}(x)=\sum_{j=1}^{\infty} a_{i j} x_{j}$ converges for each $i \in \mathbb{N}$. Throughout the paper, by $t_{k n}(A x)$, we mean

$$
t_{k n}(A x)=\frac{A_{n}(x)+A_{\sigma^{1}(n)}(x)+\cdots, A_{\sigma^{k}(n)}(x)}{k+1}, \text { for all } k, n \in \mathbb{N}
$$

A sequence space $X$ is called as solid (or normal) if $\left(\alpha_{k} x_{k}\right) \in X$ whenever $\left(x_{k}\right) \in X$ and $\left(\alpha_{k}\right)$ is a sequence of scalars such that $\left|\alpha_{k}\right| \leq 1$ for all $k \in \mathbb{N}$.

Let $X$ be a sequence space and $K=\left\{k_{1}<k_{2}<\cdots\right\} \subseteq \mathbb{N}$. The sequence space $Z_{K}^{X}=\left\{\left(x_{k n}\right) \in w:\left(x_{n}\right) \in X\right\}$ is called $K$-step space of $X$. 
A canonical preimage of a sequence $\left(x_{k n}\right) \in Z_{K}^{X}$ is a sequence $\left(y_{n}\right) \in w$ defined by

$$
y_{n}= \begin{cases}x_{n}, & \text { if } n \in \mathbb{N} \\ 0, & \text { otherwise. }\end{cases}
$$

A sequence space $X$ is monotone if it contains the canonical preimages of all its step spaces.

An Orlicz function $M$ is a function, which is continuous, non-decreasing and convex with $M(0)=0, M(x)>0$ for $x>0$ and $M(x) \longrightarrow \infty$ as $x \longrightarrow \infty$.

Lindenstrauss and Tzafriri [10] used the idea of Orlicz function to define the following sequence space. Let $w$ be the space of all real or complex sequences $x=\left(x_{k}\right)$, then

$$
\ell_{M}=\left\{x \in w: \sum_{k=1}^{\infty} M\left(\frac{\left|x_{k}\right|}{\rho}\right)<\infty\right\}
$$

which is called as an Orlicz sequence space. The space $\ell_{M}$ is a Banach space with the norm

$$
\|x\|=\inf \left\{\rho>0: \sum_{k=1}^{\infty} M\left(\frac{\left|x_{k}\right|}{\rho}\right) \leq 1\right\} .
$$

It is shown in [10] that every Orlicz sequence space $\ell_{M}$ contains a subspace isomorphic to $\ell_{p}(p \geq 1)$. The $\Delta_{2}$-condition is equivalent to $M(L x) \leq k L M(x)$ for all values of $x \geq 0$, and for $L>1$.

A sequence $\mathcal{M}=\left(M_{k}\right)$ of Orlicz function is called a Musielak-Orlicz function see ([13],[20]). A sequence $\mathcal{N}=\left(N_{k}\right)$ defined by

$$
N_{k}(v)=\sup \left\{|v| u-\left(M_{k}\right): u \geq 0\right\}, k=1,2, \cdots
$$

is called the complementary function of a Musielak-Orlicz function $\mathcal{M}$. For a given Musielak-Orlicz function $\mathcal{M}$, the Musielak-Orlicz sequence space $t_{\mathcal{M}}$ and its subspace $h_{\mathcal{M}}$ are defined as follows

$$
\begin{gathered}
t_{\mathcal{M}}=\left\{x \in w: I_{\mathcal{M}}(c x)<\infty \text { for some } c>0\right\}, \\
h_{\mathcal{M}}=\left\{x \in w: I_{\mathcal{M}}(c x)<\infty \text { for all } c>0\right\},
\end{gathered}
$$

where $I_{\mathcal{M}}$ is a convex modular defined by

$$
I_{\mathcal{M}}(x)=\sum_{k=1}^{\infty} M_{k}\left(x_{k}\right), x=\left(x_{k}\right) \in t_{\mathcal{M}} .
$$

We consider $t_{\mathcal{M}}$ equipped with the Luxemburg norm

$$
\|x\|=\inf \left\{k>0: I_{\mathcal{M}}\left(\frac{x}{k}\right) \leq 1\right\}
$$

or equipped with the Orlicz norm

$$
\|x\|^{0}=\inf \left\{\frac{1}{k}\left(1+I_{\mathcal{M}}(k x)\right): k>0\right\} .
$$

For more details about sequence spaces defined by Orlicz function see ([22], [23], [24]) and reference therein. The concept of 2-normed spaces was initially developed by Gähler[3] in the mid of 1960's, while that of 
$n$-normed spaces one can see in Misiak [14]. Since then, many others have studied this concept and obtained various results, see Gunawan $([4],[5])$ and Gunawan and Mashadi [6]. Let $n \in \mathbb{N}$ and $X$ be a linear space over the field $\mathbb{K}$, where $\mathbb{K}$ is field of real or complex numbers of dimension $d$, where $d \geq n \geq 2$. A real valued function $\|\cdot, \cdots, \cdot\|$ on $X^{n}$ satisfying the following four conditions:

(1) $\left\|x_{1}, x_{2}, \cdots, x_{n}\right\|=0$ if and only if $x_{1}, x_{2}, \cdots, x_{n}$ are linearly dependent in $X$;

(2) $\left\|x_{1}, x_{2}, \cdots, x_{n}\right\|$ is invariant under permutation;

(3) $\left\|\alpha x_{1}, x_{2}, \cdots, x_{n}\right\|=|\alpha|\left\|x_{1}, x_{2}, \cdots, x_{n}\right\|$ for any $\alpha \in \mathbb{K}$, and

(4) $\left\|x+x^{\prime}, x_{2}, \cdots, x_{n}\right\| \leq\left\|x, x_{2}, \cdots, x_{n}\right\|+\left\|x^{\prime}, x_{2}, \cdots, x_{n}\right\|$

is called a $n$-norm on $X$, and the pair $(X,\|\cdot, \cdots, \cdot\|)$ is called a $n$-normed space over the field $\mathbb{K}$.

For example, we may take $X=\mathbb{R}^{n}$ being equipped with the Euclidean $n$-norm $\left\|x_{1}, x_{2}, \cdots, x_{n}\right\|_{E}=$ the volume of the $n$-dimensional parallelopiped spanned by the vectors $x_{1}, x_{2}, \cdots, x_{n}$ which may be given explicitly by the formula

$$
\left\|x_{1}, x_{2}, \cdots, x_{n}\right\|_{E}=\left|\operatorname{det}\left(x_{i j}\right)\right|
$$

where $x_{i}=\left(x_{i 1}, x_{i 2}, \cdots, x_{i n}\right) \in \mathbb{R}^{n}$ for each $i=1,2, \cdots, n$. Let $(X,\|\cdot, \cdots, \cdot\|)$ be a $n$-normed space of dimension $d \geq n \geq 2$ and $\left\{a_{1}, a_{2}, \cdots, a_{n}\right\}$ be linearly independent set in $X$. Then the following function $\|\cdot, \cdots, \cdot\|_{\infty}$ on $X^{n-1}$ defined by

$$
\left\|x_{1}, x_{2}, \cdots, x_{n-1}\right\|_{\infty}=\max \left\{\left\|x_{1}, x_{2}, \cdots, x_{n-1}, a_{i}\right\|: i=1,2, \cdots, n\right\}
$$

defines an $(n-1)$-norm on $X$ with respect to $\left\{a_{1}, a_{2}, \cdots, a_{n}\right\}$.

A sequence $\left(x_{k}\right)$ in a $n$-normed space $(X,\|\cdot, \cdots, \cdot\|)$ is said to converge to some $L \in X$ if

$$
\lim _{k \rightarrow \infty}\left\|x_{k}-L, z_{1}, \cdots, z_{n-1}\right\|=0 \text { for every } z_{1}, \cdots, z_{n-1} \in X
$$

A sequence $\left(x_{k}\right)$ in a $n$-normed space $(X,\|\cdot, \cdots, \cdot\|)$ is said to be Cauchy if

$$
\lim _{k, p \rightarrow \infty}\left\|x_{k}-x_{p}, z_{1}, \cdots, z_{n-1}\right\|=0 \text { for every } z_{1}, \cdots, z_{n-1} \in X
$$

If every cauchy sequence in $X$ converges to some $L \in X$, then $X$ is said to be complete with respect to the $n$-norm. Any complete $n$-normed space is said to be $n$-Banach space.

In the present paper, we define some new sequence spaces by using the concept of ideal convergence, invariant mean, Musielak-Orlicz function, $n$-normed and $A$ transform as follows:

$\mathcal{I}-c_{0}^{\sigma}(A, \mathcal{M}, p,\|\cdot, \cdots, \cdot\|)=$

$$
\left\{x \in w:\left\{k \in \mathbb{N}:\left[M_{k}\left(\left\|\frac{t_{k n}(A(x))}{\rho}, z_{1}, \cdots, z_{n-1}\right\|\right)\right]^{p_{k}} \geq \epsilon\right\} \in \mathcal{I}, \text { for all } n \in \mathbb{N}\right\}
$$


$\mathcal{I}-c^{\sigma}(A, \mathcal{M}, p,\|\cdot, \cdots, \cdot\|)=$

$\left\{x \in w:\left\{k \in \mathbb{N}:\left[M_{k}\left(\left\|\frac{t_{k n}(A(x)-L)}{\rho}, z_{1}, \cdots, z_{n-1}\right\|\right)\right]^{p_{k}} \geq \epsilon\right\} \in \mathcal{I}\right.$, for all $n \in \mathbb{N}$ \& for some $\left.L \in \mathbb{C}\right\}$,

$\mathcal{I}-\ell_{\infty}^{\sigma}(A, \mathcal{M}, p,\|\cdot, \cdots, \cdot\|)=$

$$
\left\{x \in w: \exists K>0 \text { such that }\left\{k \in \mathbb{N}:\left[M_{k}\left(\left\|\frac{t_{k n}(A(x))}{\rho}, z_{1}, \cdots, z_{n-1}\right\|\right)\right]^{p_{k}} \geq K\right\} \in \mathcal{I}, \text { for all } n \in \mathbb{N}\right\} .
$$

If we take $p=\left(p_{k}\right)=1$, we get the spaces

$\mathcal{I}-c_{0}^{\sigma}(A, \mathcal{M},\|\cdot, \cdots, \cdot\|)=$

$$
\left\{x \in w:\left\{k \in \mathbb{N}:\left[M_{k}\left(\left\|\frac{t_{k n}(A(x))}{\rho}, z_{1}, \cdots, z_{n-1}\right\|\right)\right] \geq \epsilon\right\} \in \mathcal{I}, \text { for all } n \in \mathbb{N}\right\},
$$

$\mathcal{I}-c^{\sigma}(A, \mathcal{M},\|\cdot, \cdots, \cdot\|)=$

$$
\left\{x \in w:\left\{k \in \mathbb{N}:\left[M_{k}\left(\left\|\frac{t_{k n}(A(x)-L)}{\rho}, z_{1}, \cdots, z_{n-1}\right\|\right)\right] \geq \epsilon\right\} \in \mathcal{I}, \text { for all } n \in \mathbb{N} \text { \& for some } L \in \mathbb{C}\right\},
$$

$\mathcal{I}-\ell_{\infty}^{\sigma}(A, \mathcal{M},\|\cdot, \cdots, \cdot\|)=$

$$
\left\{x \in w: \exists K>0 \text { such that }\left\{k \in \mathbb{N}:\left[M_{k}\left(\left\|\frac{t_{k n} A(x)}{\rho}, z_{1}, \cdots, z_{n-1}\right\|\right)\right] \geq K\right\} \in \mathcal{I} \text {, for all } n \in \mathbb{N}\right\} .
$$

The following inequality will be used throughout the paper. If $0 \leq p_{k} \leq \sup p_{k}=H, D=\max \left(1,2^{H-1}\right)$ then

$$
\left|a_{k}+b_{k}\right|^{p_{k}} \leq D\left\{\left|a_{k}\right|^{p_{k}}+\left|b_{k}\right|^{p_{k}}\right\}
$$

for all $k$ and $a_{k}, b_{k} \in \mathbb{C}$. Also $|a|^{p_{k}} \leq \max \left(1,|a|^{H}\right)$ for all $a \in \mathbb{C}$.

The main goal of this paper is to introduce the sequence spaces $\mathcal{I}-c_{0}^{\sigma}(A, \mathcal{M}, p,\|\cdot, \cdots, \cdot\|), \mathcal{I}-c^{\sigma}(A, \mathcal{M}, p,\|\cdot, \cdots, \cdot\|)$ and $\mathcal{I}-\ell_{\infty}^{\sigma}(A, \mathcal{M}, p,\|\cdot, \cdots, \cdot\|)$ defined by a Musielak-Orlicz function $\mathcal{M}=\left(M_{k}\right)$ over $n$-normed spaces. We also make an effort to study some topological properties and prove some inclusion relation between these spaces.

\section{Main Results}

Theorem 2.1 Let $\mathcal{M}=\left(M_{k}\right)$ be a Musielak-Orlicz function, $p=\left(p_{k}\right)$ be a bounded sequence of positive real numbers. Then the spaces $\mathcal{I}-c_{0}^{\sigma}(A, \mathcal{M}, p,\|\cdot, \cdots, \cdot\|), \mathcal{I}-c^{\sigma}(A, \mathcal{M}, p,\|\cdot, \cdots, \cdot\|)$ and $\mathcal{I}-$ $\ell_{\infty}^{\sigma}(A, \mathcal{M}, p,\|\cdot, \cdots, \cdot\|)$ are linear.

Proof. Let $x, y \in \mathcal{I}-c_{0}^{\sigma}(A, \mathcal{M}, p,\|\cdot, \cdots, \cdot\|)$ and let $\alpha, \beta$ be scalars. Then there exist positive numbers $\rho_{1}$ and $\rho_{2}$ such that for every $\epsilon>0$

$$
\begin{aligned}
& D_{1}=\left\{k \in \mathbb{N}:\left[M_{k}\left(\left\|\frac{t_{k n}(A(x))}{\rho_{1}}, z_{1}, \cdots, z_{n-1}\right\|\right)\right]^{p_{k}} \geq \frac{\epsilon}{2 D}\right\} \in \mathcal{I}, \\
& D_{1}=\left\{k \in \mathbb{N}:\left[M_{k}\left(\left\|\frac{t_{k n}(A(y))}{\rho_{2}}, z_{1}, \cdots, z_{n-1}\right\|\right)\right]^{p_{k}} \geq \frac{\epsilon}{2 D}\right\} \in \mathcal{I},
\end{aligned}
$$


Let $\rho_{3}=\max \left\{2|\alpha| \rho_{1}, 2|\beta| \rho_{2}\right\}$. Since $\mathcal{M}=\left(M_{k}\right)$ is non-decreasing, convex function and so by using inequality (1.1), we have

$$
\begin{aligned}
& {\left[M_{k}\left(\left\|\frac{t_{k n}(A(\alpha x+\beta y))}{\rho_{3}}, z_{1}, \cdots, z_{n-1}\right\|\right)\right]^{p_{k}}} \\
& \quad \leq\left[M_{k}\left(\left\|\frac{t_{k n}(\alpha A(x))}{\rho_{3}}, z_{1}, \cdots, z_{n-1}\right\|\right)\right]^{p_{k}}+\left[M_{k}\left(\left\|\frac{t_{k n}(\beta A(y))}{\rho_{3}}, z_{1}, \cdots, z_{n-1}\right\|\right)\right]^{p_{k}} \\
& \quad \leq\left[M_{k}\left(\left\|\frac{t_{k n}(A(x))}{\rho_{1}}, z_{1}, \cdots, z_{n-1}\right\|\right)\right]^{p_{k}}+\left[M_{k}\left(\left\|\frac{t_{k n}(A(y))}{\rho_{2}}, z_{1}, \cdots, z_{n-1}\right\|\right)\right]^{p_{k}}
\end{aligned}
$$

Now by (2.1) and (2.2), we have

$$
\left\{k \in \mathbb{N}:\left[M_{k}\left(\left\|\frac{t_{k n}(A(\alpha x+\beta y))}{\rho_{3}}, z_{1}, \cdots, z_{n-1}\right\|\right)\right]^{p_{k}}>\epsilon\right\} \subset D_{1} \cup D_{2} .
$$

Therefore $\alpha x+\beta y \in \mathcal{I}-c_{0}^{\sigma}(A, \mathcal{M}, p,\|\cdot, \cdots, \cdot\|)$. Hence $\mathcal{I}-c_{0}^{\sigma}(A, \mathcal{M}, p,\|\cdot, \cdots, \cdot\|)$ is a linear space. Similarly we can prove that $\mathcal{I}-c^{\sigma}(A, \mathcal{M}, p,\|\cdot, \cdots, \cdot\|)$ and $\mathcal{I}-\ell_{\infty}^{\sigma}(A, \mathcal{M}, p,\|\cdot, \cdots, \cdot\|)$ are linear spaces.

Theorem 2.2 Let $\mathcal{M}=\left(M_{k}\right)$ be a Musielak-Orlicz function. Then

$$
\mathcal{I}-c_{0}^{\sigma}(A, \mathcal{M}, p,\|\cdot, \cdots, \cdot\|) \subset \mathcal{I}-c^{\sigma}(A, \mathcal{M}, p,\|\cdot, \cdots, \cdot\|) \subset \mathcal{I}-\ell_{\infty}^{\sigma}(A, \mathcal{M}, p,\|\cdot, \cdots, \cdot\|)
$$

Proof. The first inclusion is obvious. For second inclusion, let $x \in \mathcal{I}-c^{\sigma}(A, \mathcal{M}, p,\|\cdot, \cdots, \cdot\|)$. Then there exists $\rho_{1}>0$ such that for every $\epsilon>0$

$$
A_{1}=\left\{k \in \mathbb{N}:\left[M_{k}\left(\left\|\frac{t_{k n}(A(x)-L)}{\rho_{1}}, z_{1}, \cdots, z_{n-1}\right\|\right)\right]^{p_{k}} \geq \epsilon\right\} \in \mathcal{I} .
$$

Let us define $\rho=2 \rho_{1}$. Since $\mathcal{M}=\left(M_{k}\right)$ is non-decreasing and convex, we have

$$
M_{k}\left(\left\|\frac{t_{k n}(A(x))}{\rho}, z_{1}, \cdots, z_{n-1}\right\|\right) \leq M_{k}\left(\left\|\frac{t_{k n}(A(x)-L)}{\rho_{1}}, z_{1}, \cdots, z_{n-1}\right\|\right)+M_{k}\left(\left\|\frac{t_{k n}(L)}{\rho_{1}}, z_{1}, \cdots, z_{n-1}\right\|\right) .
$$

Suppose that $k \notin A_{1}$. Hence by above inequality and (1.1), we have

$$
\begin{aligned}
& {\left[M_{k}\left(\left\|\frac{t_{k n}(A(x))}{\rho}, z_{1}, \cdots, z_{n-1}\right\|\right)\right]^{p_{k}}} \\
& \quad \leq D\left\{\left[M_{k}\left(\left\|\frac{t_{k n}(A(x)-L)}{\rho_{1}}, z_{1}, \cdots, z_{n-1}\right\|\right)\right]^{p_{k}}+\left[M_{k}\left(\left\|\frac{t_{k n}(L)}{\rho_{1}}, z_{1}, \cdots, z_{n-1}\right\|\right)\right]^{p_{k}}\right\} \\
& <D\left\{\epsilon+\left[M_{k}\left(\left\|\frac{t_{k n}(L)}{\rho}, z_{1}, \cdots, z_{n-1}\right\|\right)\right]^{p_{k}}\right\} .
\end{aligned}
$$

Because of the fact that $\left[M_{k}\left(\left\|\frac{t_{k n}(L)}{\rho_{1}}, z_{1}, \cdots, z_{n-1}\right\|\right)\right]^{p_{k}} \leq \max \left\{1,\left[M_{k}\left(\left\|\frac{t_{k n}(L)}{\rho_{1}}, z_{1}, \cdots, z_{n-1}\right\|\right)\right]^{H}\right\}$, we have

$$
\left[M_{k}\left(\left\|\frac{t_{k n}(L)}{\rho}, z_{1}, \cdots, z_{n-1}\right\|\right)\right]^{p_{k}}<\infty
$$


Put $K=D\left\{\epsilon+\left[M_{k}\left(\left\|\frac{t_{k n}(L)}{\rho}, z_{1}, \cdots, z_{n-1}\right\|\right)\right]^{p_{k}}\right\}$. It follows that

$$
\left\{k \in \mathbb{N}:\left[M_{k}\left(\left\|\frac{t_{k n}(A(x))}{\rho}, z_{1}, \cdots, z_{n-1}\right\|\right)\right]^{p_{k}}>K\right\} \in \mathcal{I}
$$

which means $x \in \mathcal{I}-\ell_{\infty}^{\sigma}(A, \mathcal{M}, p,\|\cdot, \cdots, \cdot\|)$. This completes the proof of the theorem.

Theorem 2.3 Let $\mathcal{M}=\left(M_{k}\right)$ be a Musielak-Orlicz function, $p=\left(p_{k}\right)$ be a bounded sequence of positive real numbers. Then $\mathcal{I}-\ell_{\infty}^{\sigma}(A, \mathcal{M}, p,\|\cdot, \cdots, \cdot\|)$ is a paranormed space with paranorm defined by

$$
g(x)=\inf \left\{\rho>0:\left[M_{k}\left(\left\|\frac{t_{k n}(A(x))}{\rho}, z_{1}, \cdots, z_{n-1}\right\|\right)\right]^{p_{k}} \leq 1\right\}
$$

Proof. It is clear that $g(x)=g(-x)$. Since $M_{k}(0)=0$, we get $g(0)=0$. Let us take $x, y \in \mathcal{I}-$ $c_{\infty}^{\sigma}(A, \mathcal{M}, p,\|\cdot, \cdots, \cdot\|)$. Let

$$
\begin{aligned}
& B(x)=\left\{\rho>0:\left[M_{k}\left(\left\|\frac{t_{k n}(A(x))}{\rho}, z_{1}, \cdots, z_{n-1}\right\|\right)\right]^{p_{k}} \leq 1\right\}, \\
& B(y)=\left\{\rho>0:\left[M_{k}\left(\left\|\frac{t_{k n}(A(y))}{\rho}, z_{1}, \cdots, z_{n-1}\right\|\right)\right]^{p_{k}} \leq 1\right\} .
\end{aligned}
$$

Let $\rho_{1} \in B(x)$ and $\rho_{2} \in B(y)$. If $\rho=\rho_{1}+\rho_{2}$, then we have

$$
\begin{aligned}
& {\left[M_{k}\left(\left\|\frac{t_{k n}(A(x+y))}{\rho}, z_{1}, \cdots, z_{n-1}\right\|\right)\right]} \\
& \quad \leq\left(\frac{\rho_{1}}{\rho_{1}+\rho_{2}}\right)\left[M_{k}\left(\left\|\frac{t_{k n}(A(x))}{\rho_{1}}, z_{1}, \cdots, z_{n-1}\right\|\right)\right]+\left[M_{k}\left(\left\|\frac{t_{k n}(A(y))}{\rho_{2}}, z_{1}, \cdots, z_{n-1}\right\|\right)\right] .
\end{aligned}
$$

Thus $\left[M_{k}\left(\left\|\frac{t_{k n}(A(x+y))}{\rho_{1}+\rho_{2}}, z_{1}, \cdots, z_{n-1}\right\|\right)\right]^{p_{k}} \leq 1$ and

$$
\begin{aligned}
g(x+y) & \leq \inf \left\{\left(\rho_{1}+\rho_{2}\right)>0: \rho_{1} \in B(x), \rho_{2} \in B(y)\right\} \\
& \leq \inf \left\{\rho_{1}>0: \rho_{1} \in B(x)\right\}+\inf \left\{\rho_{2}>0: \rho_{2} \in B(y)\right\} \\
& =g(x)+g(y) .
\end{aligned}
$$

Let $\eta^{s} \rightarrow \eta$ where $\eta, \eta^{s} \in \mathbb{C}$ and let $g\left(x^{s}-x\right) \rightarrow 0$ as $s \rightarrow \infty$. We have to show that $g\left(\eta^{s} x^{s}-\eta x\right) \rightarrow 0$ as $s \rightarrow \infty$. Let

$$
\begin{gathered}
B\left(x^{s}\right)=\left\{\rho_{s}>0:\left[M\left(\left\|\frac{t_{k n}\left(A\left(x^{s}\right)\right)}{\rho_{s}}, z_{1}, \cdots, z_{n-1}\right\|\right)\right]^{p_{k}} \leq 1\right\}, \\
B\left(x^{s}-x\right)=\left\{\rho_{s}^{\prime}>0:\left[M\left(\left\|\frac{t_{k n}\left(A\left(x^{s}-x\right)\right)}{\rho_{s}^{\prime}}, z_{1}, \cdots, z_{n-1}\right\|\right)\right]^{p_{k}} \leq 1\right\} .
\end{gathered}
$$


If $\rho_{s} \in B\left(x^{s}\right)$ and $\rho_{s}^{\prime} \in B\left(x^{s}-x\right)$ then we observe that

$$
\begin{aligned}
{\left[M _ { k } \left(\| \frac{t_{k n}\left(A\left(\eta^{s} x^{s}-\eta x\right)\right)}{\rho_{s}\left|\eta^{s}-\eta\right|+\rho_{s}^{\prime}|\eta|}\right.\right.} & \left., z_{1}, \cdots, z_{n-1}||\right) \\
& \leq\left[M_{k}\left(|| \frac{t_{k n}\left(A\left(\eta^{s} x^{s}-\eta x^{s}\right)\right)}{\rho_{s}\left|\eta^{s}-\eta\right|+\rho_{s}^{\prime}|\eta|}+\frac{\left|\left(\eta x^{s}-\eta x\right)\right|}{\rho_{s}\left|\eta^{s}-\eta\right|+\rho_{s}^{\prime}|\eta|}, z_{1}, \cdots, z_{n-1} \|\right)\right] \\
& \leq \frac{\left|\eta^{s}-\eta\right| \rho_{s}}{\rho_{s}\left|\eta^{s}-\eta\right|+\rho_{s}^{\prime}|\eta|}\left[M_{k}\left(\left\|\frac{t_{k n}\left(A\left(x^{s}\right)\right)}{\rho_{s}}, z_{1}, \cdots, z_{n-1}\right\|\right)\right] \\
& +\frac{|\eta| \rho_{s}^{\prime}}{\rho_{s}\left|\eta^{s}-\eta\right|+\rho_{s}^{\prime}|\eta|}\left[M_{k}\left(\left\|\frac{t_{k n}\left(A\left(x^{s}-x\right)\right)}{\rho_{s}^{\prime}}, z_{1}, \cdots, z_{n-1}\right\|\right)\right.
\end{aligned}
$$

From the above inequality, it follows that

$$
\left[M_{k}\left(\left\|\frac{t_{k n}\left(A\left(\eta^{s} x^{s}-\eta x\right)\right)}{\rho_{s}\left|\eta^{s}-\eta\right|+\rho_{s}^{\prime}|\eta|}, z_{1}, \cdots, z_{n-1}\right\|\right)\right]^{p_{k}} \leq 1
$$

and consequently,

$$
\begin{aligned}
g\left(\eta^{s} x^{s}-\eta x\right) & \leq \inf \left\{\left(\rho_{s}\left|\eta^{s}-\eta\right|+\rho_{s}^{\prime}|\eta|\right)>0: \rho_{s} \in B\left(x^{s}\right), \rho_{s}^{\prime} \in B\left(x^{s}-x\right)\right\} \\
& \leq\left(\left|\eta^{s}-\eta\right|\right)>0 \inf \left\{\rho>0: \rho_{s} \in B\left(x^{s}\right)\right\} \\
& +(|\eta|)>0 \inf \left\{\left(\rho_{s}^{\prime}\right)^{\frac{p_{n}}{H}}: \rho_{s}^{\prime} \in B\left(x^{s}-x\right)\right\} \\
& \longrightarrow 0 \text { as } s \longrightarrow \infty
\end{aligned}
$$

This completes the proof of the theorem.

Theorem 2.4 Let $\mathcal{M}^{\prime}=\left(M_{k}^{\prime}\right)$ and $\mathcal{M}^{\prime \prime}=\left(M_{k}^{\prime \prime}\right)$ are Musielak-Orlicz functions that satisfies the $\Delta_{2}$ condition. Then

(i) $\mathcal{I}-c_{0}^{\sigma}\left(A, \mathcal{M}^{\prime}, p,\|\cdot, \cdots, \cdot\|\right) \subseteq \mathcal{I}-c_{0}^{\sigma}\left(A, \mathcal{M}^{\prime} \circ \mathcal{M}^{\prime \prime}, p,\|\cdot, \cdots, \cdot\|\right)$

(ii) $\mathcal{I}-c^{\sigma}\left(A, \mathcal{M}^{\prime}, p,\|\cdot, \cdots, \cdot\|\right) \subseteq \mathcal{I}-c^{\sigma}\left(A, \mathcal{M}^{\prime} \circ \mathcal{M}^{\prime \prime}, p,\|\cdot, \cdots, \cdot\|\right)$

(iii) $\mathcal{I}-l_{\infty}^{\sigma}\left(A, \mathcal{M}^{\prime}, p,\|\cdot, \cdots, \cdot\|\right) \subseteq \mathcal{I}-l_{\infty_{\theta}}^{\sigma}\left(A, \mathcal{M}^{\prime} \circ \mathcal{M}^{\prime \prime}, p,\|\cdot, \cdots, \cdot\|\right)$

Proof. (i) We prove the theorem in two parts. Firstly, let $M_{k}^{\prime}\left(\left\|\frac{t_{k n}(A(x))}{\rho}, z_{1}, \cdots, z_{n-1}\right\|\right)>\delta$. Since $\mathcal{M}^{\prime}$ is nondecreasing, convex and satisfies $\Delta_{2}$-condition, we have

$$
\begin{aligned}
{\left[M _ { k } ^ { \prime \prime } \left(M _ { k } ^ { \prime } \left(\| \frac{t_{k n}(A(x))}{\rho},\right.\right.\right.} & \left.\left.\left.z_{1}, \cdots, z_{n-1} \|\right)\right)\right]^{p_{k}} \\
& \leq\left(K \delta^{-1} M_{2}^{\prime \prime}(2)^{p_{k}}\right)\left[M_{k}^{\prime}\left(\left\|\frac{t_{k n}(A x)}{\rho}, z_{1}, \cdots, z_{n-1}\right\|\right)\right]^{p_{k}} \\
& \leq \max \left\{1,\left(K \delta^{-1} M_{k}^{\prime \prime}(2)^{H}\right)\right)^{H}\left[M_{k}^{\prime}\left(\| \frac{t_{k n}(A x)}{\rho}, z_{1}, \cdots, z_{n-1}\right)\right]^{p_{k}},
\end{aligned}
$$


where $K \geq 1$ and $\delta<1$. From the last inequality, the inclusion

$$
\begin{aligned}
\left\{k \in \mathbb{N}:\left[M_{k}^{\prime \prime}\left(M_{k}^{\prime}\left(\left\|\frac{t_{k n}(A x)}{\rho}, z_{1}, \cdots, z_{n-1}\right\|\right)\right)\right]^{p_{k}} \geq \epsilon\right\} & \\
& \subseteq\left\{k \in \mathbb{N}:\left[M_{k}^{\prime}\left(\| \frac{t_{k n}(A x)}{\rho}, z_{1}, \cdots, z_{n-1}\right)\right]^{p_{k}}\right. \\
& \left.\geq \frac{\epsilon}{\max \left\{1,\left(K \delta^{-1} M_{k}^{\prime \prime}(2)^{H}\right)\right\}}\right\}
\end{aligned}
$$

is obtained. If $x \in \mathcal{I}-c_{0}^{\sigma}\left(\mathcal{M}^{\prime}, A, p, \| \cdot, \cdots, \cdot\right)$, then the set in the right side of the above inclusion belongs to the ideal and so

$$
\left\{k \in \mathbb{N}:\left[M_{k}^{\prime \prime}\left(M_{k}^{\prime}\left(\| \frac{t_{k n}(A x)}{\rho}, z_{1}, \cdots, z_{n-1 \mid} \mid\right)\right)\right]^{p_{k}} \geq \epsilon\right\} \in \mathcal{I} .
$$

Secondly, suppose that $M_{k}^{\prime}\left(\left\|\frac{t_{k n}(A x)}{\rho}, z_{1}, \cdots, z_{n-1}\right\|\right) \leq \delta$. Since $M_{k}^{\prime \prime}$ is continuous, we have

$$
M_{k}^{\prime \prime}\left(M_{k}^{\prime}\left(\left\|\frac{t_{k n}(A x)}{\rho}, z_{1}, \cdots, z_{n-1}\right\|\right)\right)<\epsilon \text { for all } \epsilon>0
$$

which implies

$$
\mathcal{I}-\lim _{k}\left[M_{k}^{\prime \prime}\left(M_{k}^{\prime}\left(\left\|\frac{t_{k n}(A x)}{\rho}, z_{1}, \cdots, z_{n-1}\right\|\right)\right)\right]^{p_{k}}=0 \quad \text { as } \epsilon \rightarrow 0 .
$$

This completes the proof of (i) part. Similarly, we can prove other parts.

Theorem 2.5 Let $\mathcal{M}^{\prime}=\left(M_{k}^{\prime}\right)$ and $\mathcal{M}^{\prime \prime}=\left(M_{k}^{\prime \prime}\right)$ are Musielak-Orlicz functions that satisfies the $\Delta_{2}$ condition. Then

(i) $\mathcal{I}-c_{0}^{\sigma}(A, \mathcal{M}, p,\|\cdot, \cdots, \cdot\|) \cap \mathcal{I}-c_{0}^{\sigma}\left(A, \mathcal{M}^{\prime}, p,\|\cdot, \cdots, \cdot\|\right) \subseteq \mathcal{I}-c_{0}^{\sigma}\left(A, \mathcal{M}^{\prime}+\mathcal{M}, p,\|\cdot, \cdots, \cdot \cdot\|\right)$

(ii) $\mathcal{I}-c^{\sigma}(A, \mathcal{M}, p,\|\cdot, \cdots, \cdot\|) \cap \mathcal{I}-c^{\sigma}\left(A, \mathcal{M}^{\prime}, p,\|\cdot, \cdots, \cdot\|\right) \subseteq \mathcal{I}-c^{\sigma}\left(A, \mathcal{M}^{\prime}+\mathcal{M}, p,\|\cdot, \cdots, \cdot\|\right)$

(iii) $\mathcal{I}-l_{\infty}^{\sigma}(A, \mathcal{M}, p,\|\cdot, \cdots, \cdot\|) \cap \mathcal{I}-l_{\infty}^{\sigma}\left(A, \mathcal{M}^{\prime}, p,\|\cdot, \cdots, \cdot\|\right) \subseteq \mathcal{I}-l_{\infty}^{\sigma}\left(A, \mathcal{M}^{\prime}+\mathcal{M}, p,\|\cdot, \cdots, \cdot\|\right)$.

Proof. (i) Let $x \in \mathcal{I}-c_{0}^{\sigma}(A, \mathcal{M}, p,\|\cdot, \cdots, \cdot\|) \cap \mathcal{I}-c_{0}^{\sigma}\left(A, \mathcal{M}^{\prime}, p,\|\cdot, \cdots, \cdot\|\right)$. Then there exists $K_{1}>0$ and $K_{2}>0$ such that

$$
A_{1}=\left\{k \in \mathbb{N}:\left[M_{k}\left(\left\|\frac{t_{k n}(A(x))}{\rho_{1}}, z_{1}, \cdots, z_{n-1}\right\|\right)\right]^{p_{k}} \geq K_{1}\right\} \in \mathcal{I}
$$

and

$$
A_{2}=\left\{k \in \mathbb{N}:\left[M_{k}^{\prime}\left(\left\|\frac{t_{k n}(A(x))}{\rho_{1}}, z_{1}, \cdots, z_{n-1}\right\|\right)\right]^{p_{k}} \geq K_{2}\right\} \in \mathcal{I}
$$

for some $\rho>0$. Let $k \notin A_{1} \cup A_{2}$. Then we have

$$
\begin{aligned}
{\left[( M _ { k } + M _ { k } ^ { \prime } ) \left(\| \frac{t_{k n}(A(x))}{\rho}, z_{1}, \cdots,\right.\right.} & \left.\left.z_{n-1} \|\right)\right]^{p_{k}} \\
& \leq D\left\{\left(M_{k}\left(\left\|\frac{t_{k n}(A(x))}{\rho}, z_{1}, \cdots, z_{n-1}\right\|\right)\right)^{p_{k}}\right. \\
& +\left(M_{k}^{\prime}\left(\left\|\frac{t_{k n}(A(x))}{\rho}, z_{1}, \cdots, z_{n-1}\right\|\right)\right)^{p_{k}} \\
& <\left\{K_{1}+K_{2}\right\} .
\end{aligned}
$$


$k \notin B=\left\{k \in \mathbb{N}:\left[\left(M_{k}^{\prime}+M_{k}\right)\left(\left\|\frac{t_{k n}(A(x))}{\rho}, z_{1}, \cdots, z_{n-1}\right\|\right)\right)^{p_{k}}>K\right\}$. We have $A_{1} \cup A_{2} \in \mathcal{I}$ and so $B \subset A_{1} \cup A_{2}$ which implies $B \in \mathcal{I}$. This means that $x \in \mathcal{I}-c_{0}^{\sigma}\left(A, \mathcal{M}^{\prime}+\mathcal{M}, p,\|\cdot, \cdots, \cdot\|\right)$. This completes the proof of (i) part of the theorem. Similarly, we can prove (ii) and (iii) part.

Theorem 2.6 If $\sup _{k}\left[M_{k}(t)\right]^{p_{k}}<\infty$ for all $t>0$, then we have

$$
\mathcal{I}-c^{\sigma}(A, \mathcal{M}, p,\|\cdot, \cdots, \cdot\|) \subseteq \mathcal{I}-\ell_{\infty}^{\sigma}(A, \mathcal{M}, p,\|\cdot, \cdots, \cdot\|) .
$$

Proof. Let $x \in \mathcal{I}-c^{\sigma}(A, \mathcal{M}, p,\|\cdot, \cdots, \cdot\|)$. By using inequality (1.1), we have

$$
\begin{aligned}
{\left[M_{k}\left(\| \frac{t_{k n}(A(x))}{\rho}\right)\right]^{p_{k}} } & \leq D\left\{\left[M_{k}\left(\left\|\frac{t_{k n}(A(x)-L)}{\rho}, z_{1}, \cdots, z_{n-1}\right\|\right)\right]^{p_{k}}\right. \\
& \left.+\left[M_{k}\left(\left\|\frac{t_{k n}(L)}{\rho}, z_{1}, \cdots, z_{n-1}\right\|\right)\right]^{p_{k}}\right\}
\end{aligned}
$$

where $\rho=2 \rho_{1}$. Hence, we have

$$
\left\{k \in \mathbb{N}:\left[M_{k}\left(\| \frac{t_{k n}(A(x))}{\rho}\right)\right]^{p_{k}} \geq K\right\} \subseteq\left\{k \in \mathbb{N}:\left[M_{k}\left(\| \frac{t_{k n}(A(x)-L)}{\rho_{1}}, z_{1}, \cdots, z_{n-1}\right)\right]^{p_{k}} \geq \epsilon\right\}
$$

for all $n$ and some $K>0$. Since the set in the right side of the above inclusion belongs to the ideal, all of its subsets are in the ideal. Hence

$$
\left\{k \in \mathbb{N}:\left[M_{k}\left(\| \frac{t_{k n}(A(x))}{\rho}\right)\right]^{p_{k}} \geq K\right\} \in \mathcal{I}
$$

which completes the proof.

Theorem 2.7 Let $0<p_{k} \leq q_{k}<\infty$ for each $k \in \mathbb{N}$ and $\left(\frac{q_{k}}{p_{k}}\right)$ be bounded. Then following inclusions hold (i) $\mathcal{I}-c_{0}^{\sigma}(A, \mathcal{M}, q,\|\cdot, \cdots, \cdot\|) \subseteq \mathcal{I}-c_{0}^{\sigma}(A, \mathcal{M}, p,\|\cdot, \cdots, \cdot\|)$

(ii) $\mathcal{I}-c^{\sigma}(A, \mathcal{M}, q,\|\cdot, \cdots, \cdot\|) \subseteq \mathcal{I}-c^{\sigma}(A, \mathcal{M}, p,\|\cdot, \cdots, \cdot \cdot\|)$.

Proof. (i) Let $x \in \mathcal{I}-c_{0}^{\sigma}(A, \mathcal{M}, q,\|\cdot, \cdots, \cdot\|)$. Write $\alpha_{k}=\frac{p_{k}}{q_{k}}$. By hypothesis, we have $0<\alpha \leq \alpha_{k} \leq 1$. If $\left[M_{k}\left(\left\|\frac{t_{k n}(A(x))}{\rho}, z_{1}, \cdots, z_{n-1}\right\|\right)\right]^{q_{k}} \geq 1$, the inequality

$$
\left[M_{k}\left(\left\|\frac{t_{k n}(A(x))}{\rho}, z_{1}, \cdots, z_{n-1}\right\|\right)\right]^{p_{k}} \leq\left[M_{k}\left(\left\|\frac{t_{k n}(A(x))}{\rho}, z_{1}, \cdots, z_{n-1}\right\|\right)\right]^{q_{k}}
$$

holds. This implies the inclusion

$$
\begin{aligned}
\left\{k \in \mathbb{N}:\left[M_{k}\left(\left\|\frac{t_{k n}(A(x))}{\rho}, z_{1}, \cdots, z_{n-1}\right\|\right)\right]^{p_{k}} \geq \epsilon\right\} \\
\quad \subseteq\left\{k \in \mathbb{N}:\left[M_{k}\left(\left\|\frac{t_{k n}(A(x))}{\rho}, z_{1}, \cdots, z_{n-1}\right\|\right)\right]^{q_{k}} \geq \epsilon\right\}
\end{aligned}
$$


and so the result is obvious. Conversely, if $\left[M_{k}\left(\left\|\frac{t_{k n}(A(x))}{\rho}, z_{1}, \cdots, z_{n-1}\right\|\right)\right]^{q_{k}}<1$, we obtain the following inclusion

$$
\begin{aligned}
\left\{k \in \mathbb{N}:\left[M_{k}\left(\left\|\frac{t_{k n}(A(x))}{\rho}, z_{1}, \cdots, z_{n-1}\right\|\right)\right]^{p_{k}} \geq \epsilon\right\} & \\
& \subseteq\left\{k \in \mathbb{N}:\left[M_{k}\left(\left\|\frac{t_{k n}(A(x))}{\rho}, z_{1}, \cdots, z_{n-1}\right\|\right)\right]^{q_{k}} \geq \epsilon^{\frac{1}{\alpha}}\right\}
\end{aligned}
$$

since then the inequality

$$
\left[M_{k}\left(\left\|\frac{t_{k n}(A(x))}{\rho}, z_{1}, \cdots, z_{n-1}\right\|\right)\right]^{p_{k}} \leq\left(\left[M_{k}\left(\left\|\frac{t_{k n}(A(x))}{\rho}, z_{1}, \cdots, z_{n-1}\right\|\right)\right]^{q_{k}}\right)^{\alpha}
$$

holds. Hence we conclude that $x \in \mathcal{I}-c_{0}^{\sigma}(A, \mathcal{M}, p,\|\cdot, \cdots, \cdot\|)$. This completes the proof of (i) part. Similarly, we can prove (ii) part.

Theorem 2.8 If $0<\inf p_{k} \leq p_{k} \leq 1$ for each $k \in \mathbb{N}$. Then the following inclusions hold:

(i) $\mathcal{I}-c_{0}^{\sigma}(A, \mathcal{M}, p,\|\cdot, \cdots, \cdot\|) \subseteq \mathcal{I}-c_{0}^{\sigma}(A, \mathcal{M},\|\cdot, \cdots, \cdot\|)$

(ii) $\mathcal{I}-c^{\sigma}(A, \mathcal{M}, p,\|\cdot, \cdots, \cdot\|) \subseteq \mathcal{I}-c^{\sigma}(A, \mathcal{M},\|\cdot, \cdots, \cdot\|)$.

Proof. Let $x \in \mathcal{I}-c_{0}^{\sigma}(A, \mathcal{M}, p,\|\cdot, \cdots, \cdot\|)$. Suppose that $k \notin\left\{\left[M_{k}\left(\| \frac{t_{k n}(A(x))}{\rho}, z_{1}, \cdots, z_{n-1}\right)\right]^{p_{k}} \geq \epsilon\right\}$ for $0<\epsilon<1$. By hypothesis, the inequality

$$
M_{k}\left(\left\|\frac{t_{k n}(A(x))}{\rho}, z_{1}, \cdots, z_{n-1}\right\|\right) \leq\left[M_{k}\left(\left\|\frac{t_{k n}(A(x))}{\rho}, z_{1}, \cdots, z_{n-1}\right\|\right)\right]^{p_{k}}
$$

holds. Then we have $k \notin\left\{k \in \mathbb{N}: M_{k}\left(\left\|\frac{t_{k n}(A(x))}{\rho}, z_{1}, \cdots, z_{n-1}\right\|\right) \geq \epsilon\right\}$ which implies

$$
\begin{aligned}
\left\{k \in \mathbb{N}: M_{k}\left(\left\|\frac{t_{k n}(A(x))}{\rho}, z_{1}, \cdots, z_{n-1}\right\|\right)\right. & \geq \epsilon\} \\
& \subseteq\left\{k \in \mathbb{N}:\left[M_{k}\left(\left\|\frac{t_{k n}(A(x))}{\rho}, z_{1}, \cdots, z_{n-1}\right\|\right)\right]^{p_{k}} \geq \epsilon\right\} .
\end{aligned}
$$

Hence $x \in \mathcal{I}-c_{0}^{\sigma}(A, \mathcal{M},\|\cdot, \cdots, \cdot\|)$ since the set

$$
\left\{k \in \mathbb{N}: M_{k}\left(\left\|\frac{t_{k n}(A(x))}{\rho}, z_{1}, \cdots, z_{n-1}\right\|\right) \geq \epsilon\right\} \in \mathcal{I}
$$

This completes the proof of (i) part. Similarly, we can prove (ii) part.

Corollary 2.9 If $0<\inf p_{k} \leq p_{k} \leq 1$ for each $k \in \mathbb{N}$. Then the following inclusions hold:

(i) $\mathcal{I}-c_{0}^{\sigma}(A, \mathcal{M},\|\cdot, \cdots, \cdot\|) \subseteq \mathcal{I}-c_{0}^{\sigma}(A, \mathcal{M}, p,\|\cdot, \cdots, \cdot\|)$

(ii) $\mathcal{I}-c^{\sigma}(A, \mathcal{M},\|\cdot, \cdots, \cdot\|) \subseteq \mathcal{I}-c^{\sigma}(A, \mathcal{M}, p,\|\cdot, \cdots, \cdot\|)$.

Proof. The proof is obvious by Theorem 2.8. 


\section{REFERENCES}

[1] P. Das, P. Kostyrko, W. Wilczynski and P. Malik, I and $I^{*}$ convergence of double sequences, Math. Slovaca, 58 (2008), 605-620.

[2] P. Das and P.Malik, On the statistical and I- variation of double sequences, Real Anal. Exch. 33 (2)(2007-2008), 351-364.

[3] S. Gähler, Linear 2-normietre Rume, Math. Nachr., 28 (1965), 1-43.

[4] H. Gunawan, On n-Inner Product, n-Norms, and the Cauchy-Schwartz Inequality, Sci. Math. Jap., 5 (2001), $47-54$.

[5] H. Gunawan, The space of p-summable sequence and its natural n-norm, Bull. Aust. Math. Soc., 64 (2001), 137-147.

[6] H. Gunawan and M. Mashadi, On n-normed spaces, Int. J. Math. Math. Sci., 27 (2001), 631-639.

[7] E. E. Kara, M. Daştan and M. Ïlkhan, On almost ideal convergence with respect to an Orlicz function, Konuralp J. Math. 4 (2016), 87-94.

[8] P. Kostyrko, T. Salat and W. Wilczynski, I-Convergence, Real Anal. Exch. 26 (2) (2000), 669-686.

[9] V. Kumar, On I and I* convergence of double sequences, Math. Commun., 12 (2007), 171-181.

[10] J. Lindenstrauss and L. Tzafriri, On Orlicz sequence spaces, Israel J. Math. 10(1971), 345-355.

[11] G. G. Lorentz, A contribution to the theory of divergent series, Acta Math. 80(1948), 167-190.

[12] I. J. Maddox, Spaces of strongly summable sequence, Q. J. Math; 18(1967), 345-355.

[13] L. Maligranda, Orlicz spaces and interpolation, Seminars in Mathematics 5, Polish Academy of Science, 1989.

[14] A. Misiak, n-inner product spaces, Math. Nachr. 140 (1989), 299-319.

[15] M. Mursaleen and A. Alotaibi, On I-convergence in radom 2-normed spaces, Math. Slovaca, 61(6)(2011), 933-940.

[16] M. Mursaleen, S. A. Mohiuddine and O. H. H. Edely, On ideal convergence of double sequences in intuitioistic fuzzy normed spaces, Comput. Math. Appl., 59 (2010), 603-611.

[17] M. Mursaleen and S. A. Mohiuddine, On ideal convergence of double sequences in probabilistic normed spaces, Math. Reports, 12(64)(4) (2010), 359-371.

[18] M. Mursaleen and S. A. Mohiuddine, On ideal convergence in probabilistic normed spaces, Math. Slovaca, 62(2012), 49-62.

[19] M. Mursaleen and S. K. Sharma, Spaces of ideal convergent sequences, World Sci. J. 2014(2014), Art. ID 134534.

[20] J. Musielak, Orlicz spaces and modular spaces, Lecture Notes in Mathematics, Springer-Verlag Berlin Heidelberg, (1983).

[21] K. Raj and S. K. Sharma, Ideal convergent sequence spaces defined by a Musielak-Orlicz function, Thai. J. Math., 11 (2013), 577-587.

[22] K. Raj and S. K. Sharma, Some sequence spaces in 2-normed spaces defined by Musielak-Orlicz functions, Acta Univ. Sapientiae Math., 3 (2011), 97-109.

[23] K. Raj and S. K. Sharma, Some generalized difference double sequence spaces defined by a sequence of Orlicz-function, Cubo, 14 (2012), 167-189.

[24] K. Raj and S. K. Sharma, Some multiplier sequence spaces defined by a Musielak-Orlicz function in n-normed spaces, N. Z. J. Math. 42 (2012), 45-56.

[25] A. Şahiner, M. Gürdal, S. Saltan and H. Gunawan, On ideal convergence in 2-normed spaces, Taiwanese J. Math., 11 (2007), 1477-1484.

[26] P. Schaefer, Invariant matrices and invariant means, Proc. Amer. Math. Soc.; 36(1972), 104-110.

[27] B. C. Tripathy and B. Hazarika, Some I-convergent sequence spaces defined by Orlicz functions, Acta Math. Appl. Sin. Engl. Ser. 27 (2011), 149-154. 